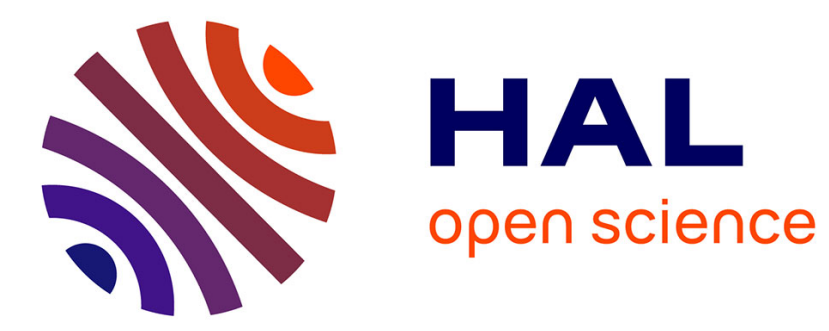

\title{
La promotion de l'animal sensible, une révolution dans la Révolution
}

Eric Baratay

\section{To cite this version:}

Eric Baratay. La promotion de l'animal sensible, une révolution dans la Révolution. Revue historique, 2012, Requérir le pouvoir, 661, pp.131-153. halshs-01569484

\section{HAL Id: halshs-01569484 \\ https://shs.hal.science/halshs-01569484}

Submitted on 26 Jul 2017

HAL is a multi-disciplinary open access archive for the deposit and dissemination of scientific research documents, whether they are published or not. The documents may come from teaching and research institutions in France or abroad, or from public or private research centers.
L'archive ouverte pluridisciplinaire HAL, est destinée au dépôt et à la diffusion de documents scientifiques de niveau recherche, publiés ou non, émanant des établissements d'enseignement et de recherche français ou étrangers, des laboratoires publics ou privés. 


\title{
La promotion de l'animal sensible. Une révolution dans la Révolution
}

\author{
Éric BARATAY
}

Les questions de la sensibilité de l'animal, de son respect et de sa protection, de l'extension du droit à son profit, de son utilisation éthique prennent une importance croissante dans notre civilisation contemporaine, notamment ces dernières années à mesure que montent les craintes sur l'avenir de la biodiversité et que l'éthologie récente révèle les richesses animales ${ }^{1}$. Pourtant, toutes ces questions ne sont pas récentes en Occident ${ }^{2}$. Bien au contraire, elles ont été quelquefois affirmées, plus souvent réfutées depuis l'Antiquité grecque. Celle-ci livre les premières traces écrites d'une réflexion concentrée sur la nature et les facultés des âmes, parce qu'elles servent à définir les êtres animés et à décider du traitement à leur accorder. La raison, promue condition de la citoyenneté et de la participation aux débats de la cité, devient l'attribut premier de l'âme humaine et le critère majeur de la distinction : sa présence place au même rang que l'homme, son absence garantit l'infériorité, et l'habitude s'impose ainsi de penser l'animal par l'homme. Alors qu'un courant philosophique minoritaire insiste sur la parenté des âmes et des facultés, au regard des comportements des uns et des autres, pour prôner respect, fraternité et refus de tuer, une majorité considère que les ressemblances des

1. Dominique Lestel, Les Origines animales de la culture, Paris, Flammarion, 2001 ; Jacques Derrida, L'Animal que donc je suis, Paris, Galilée, 2006 ; Yves Christen, L'animal est-il une personne?, Paris, Flammarion, 2009.

2. Pour un panorama historique de la condition animale, voir Éric Baratay, Et l'homme créa l'animal, histoire d'une condition, Paris, Odile Jacob, 2003 ; Linda Kalof, Brigitte Resl (dir.), A Cultural History of Animals, Oxford, Berg Publishers, 2007. 
actes extérieurs ne prouvent rien, soutient que seul l'homme possède une âme raisonnable et qu'il existe une différence de nature interdisant toute déférence vis-à-vis des bêtes, toute communauté de droit et de justice ${ }^{3}$.

Ce débat est introduit dans le monde romain, à partir du ${ }^{\text {er }}$ siècle avant J.-C., mais la christianisation le réduit peu à peu. L'opposition à la métempsycose et au végétarisme prônés par des sectes païennes conduit l'Église à assimiler au paganisme puis à l'hérésie les idées favorables aux animaux et à les combattre. À l'inverse, elle utilise certaines philosophies grecques, notamment celles de Platon et d'Aristote, pour interpréter une Bible en réalité très vague sur les différences entre l'homme et l'animal comme le montre l'exégèse actuelle ${ }^{4}$, pour bâtir une synthèse entre la foi et la raison, pour imposer la thèse d'une rupture entre l'homme et l'animal. Alors que le premier est doté d'une âme spirituelle, immortelle, raisonnable, le second est réduit aux facultés limitées, instinctives et sensitives d'une âme corporelle et mortelle. Renvoyé dans le profane et le matériel, l'animal est bon à exploiter. Sa sensibilité n'est pas niée, même si elle est quelquefois minorée, mais elle est jugée sans importance. Cette position fait la quasi-unanimité jusqu'au $\mathrm{XVI}^{\mathrm{e}}$ siècle (bien que des marginaux, comme François d'Assise, ne l'adoptent pas), car les philosophes sont alors des clercs et la philosophie est une théologie ${ }^{5}$.

Le débat renaît aux $\mathrm{XVI}^{\mathrm{e}}$-XVIII ${ }^{\mathrm{e}}$ siècles dans un contexte nouveau : le développement de l'imprimerie et de l'humanisme permet la redécouverte de penseurs grecs et de thèses oubliées; la révolution scientifique met en cause la place centrale de l'homme, insiste sur la parenté avec les autres vivants; tout cela favorise une montée de l'esprit critique, une discussion des idées et des dogmes. Ainsi, le XVI siècle voit la renaissance de la notion, initiée par Plutarque, de communauté des êtres au nom d'un partage de la raison. Elle est notamment adoptée par Montaigne, affirmant qu'elle devrait obliger l'homme à la bienveillance et au "devoir d'humanité ". Mais, comme en Grèce antique, cette promotion de l'animal est mal accueillie, perçue

3. Voir Élisabeth de Fontenay, Plutarque: trois traités sur les animaux, précédé de La Raison du plus fort, Paris, POL, 1992 ; Thierry Gontier, L'Homme et l'animal, la philosophie antique, Paris, PUF, 1999 ; Jacques Dumont, Les Animaux dans l'antiquité grecque, Paris, L'Harmattan, 2003.

4. Albert de Pury, Homme et animal Dieu les créa. L'Ancien Testament et les animaux, Paris, Labor et Fides, 1993.

5. Voir la synthèse de Éric Baratay, "L'anthropocentrisme du christianisme occidental », dans Si les lions pouvaient parler. Essais sur la condition animale, Boris Cyrulnik (dir.), Paris, 1998, p. 1428-1449, et les travaux de Jacques Voisenet, Bestiaire chrétien. Limagerie animale des auteurs du Haut Moyen Age, Toulouse, Presses universitaires du Mirail, 1994, et Bêtes et hommes dans le monde médiéval, le bestiaire des clercs, Louvain, Brépols, 2000 ; Éric Baratay, L'Église et l'animal (France, XVIr$X X^{*}$ siècle), Paris, Cerf, 1996. 
comme une déstabilisation sociale, l'homme disant avoir besoin des animaux pour vivre, d'où la réticence, jusqu'à nos jours, à réviser leur portrait d'êtres irraisonnables et instinctifs, justifiant leur traitement. C'est ainsi que la contestation humaniste suscite la contre-philosophie la plus radicale avec Descartes : il n'existe que des corps matériels et des âmes immortelles; il est impensable que les animaux possèdent de telles âmes; ce ne sont que des machines ne connaissant par la douleur, utilisables à volonté.

Cette idée de l'animal-machine est refusée par les philosophes du XVIII $^{\mathrm{e}}$ siècle au titre de son invraisemblance. Ils s'appuient sur Locke soutenant que les idées ne sont pas innées mais se forment par l'expérience et par les sens. Ils en déduisent que l'âme animale est de même nature que l'humaine, matérielle pour les uns, tel Diderot, spirituelle pour les autres, tels Condillac, Voltaire, Rousseau, mais avec des différences de degré faisant que l'animal possède la sensibilité, l'émotion, le langage, le raisonnement concret, la capacité d'adaptation mais pas la réflexion, la parole, la connaissance intellectuelle ou l'inquiétude métaphysique. Ce portrait sert plus à distinguer l'homme de l'animal qu'à connaître celui-ci et il conduit à des positions divergentes sur le traitement licite : pour les uns, de simples différences de degré exigent le respect de l'animal ; pour d'autres, elles sont suffisamment importantes pour autoriser l'indifférence. ${ }^{6}$. En fait, le débat ne porte pas directement sur le traitement de l'animal, seulement abordé comme une conséquence des positions théoriques, et la controverse reste de nature intellectuelle, philosophique, religieuse, par livres interposés. Il n'empêche qu'elle irradie la société cultivée du XVIII ${ }^{e}$ siècle, comme bien d'autres débats des Lumières, et qu'elle prépare ainsi le bouleversement révolutionnaire.

En effet, la Révolution permet l'éclosion d'un débat public, aux deux sens du terme, c'est-à-dire organisé dans des lieux publics et avec la participation d'un public cultivé, pas seulement de quelques penseurs. Il porte directement sur la question des traitements quotidiens infligés aux animaux et prend ainsi un caractère social et politique. Il est créé et rendu possible par le contexte révolutionnaire de relecture du monde, remettant en cause diverses conditions, des sujets aux esclaves, des femmes aux enfants, et s'étendant jusqu'aux animaux

6. Voir Thierry Gontier, De l'animal à l'homme. Montaigne et Descartes ou les paradoxes de la philosophie moderne sur la nalure des animaux, Paris, Vrin, 1998, et (dir.), Animal et animalité dans la philosophie de la Renaissance et de l'age classique, Louvain, Peeters, 2005 ; Elisabeth de Fontenay, Le Silence des bêtes. La philosophie à l'épreuve de l'animalité, Paris, Fayard, 1998 ; Jean-Luc Guichet, Rousseau, l'animal et l'homme: l'animalité dans l'horizon anthropologique des Lumières, Paris, Cerf, 2006, et (dir.), De l'animal machine à l'âme des machines, querelles biomécaniques de l'âme (XVIr-XXT), Paris, PL Sorbonne, 2010, et avec Jacques Berchtold (dir.), L'Animal des Lumières, numéro spécial Dix-huitième siècle, $42,2010,1$. 
par cette volonté de tout mettre à bas et de reconstruire. De ce fait, il est nourri de prises de position, non pas vraiment nouvelles mais radicales, condamnant les manières habituelles de traiter les bêtes, proposant de nouvelles relations entre une humanité nouvelle et une nouvelle animalité. Par cette volonté de penser autrement, par cette radicalité, il annonce le chamboulement intellectuel, culturel et juridique du dernier tiers du $\mathrm{XX}^{\mathrm{e}}$ siècle et des débuts du XXI ${ }^{\mathrm{e}}$ siècle à propos des relations entre les hommes et les animaux.

\section{DES LIEUX ET DES HOMMES}

Ce débat, ces positions prennent corps çà et là, à l'occasion de projets, de concours, de discours officiels, dont voici trois exemples significatifs. Le premier concerne le transfert de la ménagerie royale de Versailles. Celle-ci est investie, saccagée, abolie par des Jacobins peu après le 10 août 1792 et la plupart des bêtes sont tuées sur place ou envoyées à la boucherie. Comme il reste quelques survivantes, un débat s'engage sur l'utilité de leur transfert à Paris, au jardin du nouveau Muséum d'histoire naturelle. L'idée est soutenue par des naturalistes persuadés de la nécessité de ménageries pour la science : elles permettraient l'étude des comportements des animaux mais aussi l'acclimatation et la domestication des bêtes exotiques. Cela favoriserait l'alimentation, les transports attelés, les travaux agricoles ou encore l'industrie textile, des aspects jugés indispensables à l'heure de la physiocratie, des Lumières, puis des restrictions de l'époque révolutionnaire. Le projet est combattu par d'autres dans le sillage de l'Encyclopédie, dont l'article « Ménagerie » avait proclamé qu'il fallait « détruire les ménageries lorsque les peuples manquent de pain ; il serait honteux de nourrir des bêtes à grands frais lorsqu'on a autour de soi des hommes qui meurent de faim ${ }^{7}$. Après des hésitations, le Comité de salut public entérine, en 1794, la création d'une ménagerie nationale au Jardin des plantes, constituée de bêtes ramassées ou réquisitionnées çà et là ${ }^{8}$. Encore faut-il décider de l'allure à lui donner, des animaux à accueillir, des buts à rechercher, des conditions

7. Neuchâtel, Faulche, 1765, t. X; un exemple de cette tendance: AN, AJ 15512 , pièces $510-511$, lettres du régisseur du domaine de Versailles, 19 septembre 1792 et 17 janvier
1793 .

8. Yves Laissus, Les Animaux du muséum, 1793-1993, Paris, Imprimerie nationale, 1993 ; Randy Burkhardt, "La ménagerie et la vie du Muséum ", dans Le Muséum au premier siècle de son histore, Claude Blanckaert et alti (dir.), Paris, 1997, p. 481-508. 
à créer, ce qui prolonge le débat et celui-ci donne lieu à plusieurs propositions imprimées, destinées aussi bien à l'opinion publique qu'au gouvernement ${ }^{9}$.

Deuxième exemple : lors de la séance publique du 6 juillet 1802, la classe des sciences morales et politiques de l'Institut national annonce le sujet de son prochain prix de morale : "Jusqu'à quel point les traitements barbares exercés sur les animaux intéressent-ils la morale publique? et conviendrait-il de faire des lois à cet égard ? " ${ }^{10}$ Les candidats doivent envoyer leurs mémoires quelques mois plus tard et le prix doit être décerné en 1803. L'usage est commun dans la France du XVIII siècle et c'est en envoyant L'Origine et les fondements de l'inégalité à l'Académie de Dijon et en recevant le premier prix d'un concours que Rousseau s'était fait connaître en 1755. Pour le concours de Paris, 27 mémoires sont déposés, deux étant ensuite imprimés par leurs auteurs en 1804 et $1805^{11}$, et cela représente un nombre assez important au regard des autres sujets discutés à l'époque.

Le troisième exemple a lieu le 21 avril 1803 lors d'une séance publique et solennelle, présidée par le préfet du Rhône, à l'École vétérinaire de Lyon. Elle a pour but de décerner plusieurs prix, d'anatomie, de matière médicale, de pathologie, aux meilleurs étudiants en fin d'études. La séance est conclue par les discours du préfet, du maire de Lyon et du professeur de matière médicale, chimie et botanique, auteur d'une allocution forte et novatrice sur les devoirs des vétérinaires envers les animaux (cf. annexe). Or, des comptes rendus de ces séances solennelles, et celle-ci n'échappe pas à la règle ${ }^{12}$, étaient régulièrement publiés, avec les textes des discours, distribués largement et amplement lus en France et en Europe du fait du prestige de cette école vétérinaire, la première créée au monde en $1761^{13}$.

9. Jacques Bernardin de Saint-Pierre, Mémoire sur la nécessité de joindre une ménagerie au Jardin national des plantes, Paris, Didot, 1792 ; Aubin Millin, Philippe Pinel, Alexandre Brongniart, Rapport fait à la Société d'histoire naturelle de Paris, Paris, Boileau, 1792 ; Étienne de Lacépède, « Lettre relative aux établissements publics destinés à renfermer des animaux vivants ", La Décade philosophique, 8, An IV [1795], p. 449-462.

10. La Décade philosophique, 34, An X [1802], p. 123-124.

11. Les mémoires sont aux archives de l'Institut, côte I H. 8, manuscrits. Les deux publiés : Joseph-Louis Grandchamp, Essai philosophique, Paris, Fain jeune, 1804 ; Jean-Baptiste Salaville, De l'homme et des animaux, Paris, Déterville, 1805, d'esprit cartésien. Le concours et les mémoires ont été étudiés pour la première fois par Valentin Pelosse, "Imaginaire social et protection de l'animal. Des amis des bêtes de l'an X au législateur de 1850 »,L'Homme, 21, 1981, p. 5-33, et 22, 1982, p. 33-51.

12. Procès-verbal de la séance publique tenue à l'École vétérinaire de Lyon, le Ir floréal an XI, Lyon, École vétérinaire de Lyon, s. d.

13. Jack Bost, Lyon, berceau des sciences véténinaire, Lyon, Éditions lyonnaises d'art et d'histoire, 1992 ; Ronald Hubscher, Les Maîtres des bêtes. Les véténnaires dans la société française, Paris, Odile Jacob, 2003. 
Derrière les occasions et les lieux, il y a des hommes. Le débat est initié par quelques personnalités, des philosophes, des écrivains, des scientifiques, notamment le naturaliste Lacépède (1756-1825), alors connu pour ses publications consacrées aux quadrupèdes, et Bernardin de Saint-Pierre (1737-1814) à la fois naturaliste, ses Études de la nature (1784) connaissent un vif succès le conduisant à l'intendance du Jardin des plantes en 1792, et écrivain. Paul et Virginie (1787), au retentissement encore plus grand, le rend célèbre en Europe et le promeut défenseur des Noirs. Bernardin de Saint-Pierre a été très proche de Buffon et de Jean-Jacques Rousseau avec qui il a eu de nombreux entretiens sur la nature dans les années 1770 . Il est à l'origine du débat sur la ménagerie de Versailles et probablement de la question du concours de 1802, puisqu'il fait partie, depuis 1795 , de la sixième section, dite "Morale", de la classe des sciences morales et politiques ${ }^{14}$, section à l'initiative du sujet sur les animaux et dans laquelle se trouvent des personnages influents de l'Institut. Par exemple, l'écrivain Louis-Sébastien Mercier (1740-1814), auteur du Tableau de Paris (1781-1788) et du Nouveau Paris (1800), fourmillant de considérations sur le sort des animaux, ainsi que d'un hommage à l'influence de Rousseau sur la Révolution (1791 $)^{15}$, mais aussi l'ancien enseignant et politique Joseph Lakanal (1762-1845), réorganisateur du Jardin du roi en Muséum national d'histoire naturelle, ou encore Louis-Marie La Revellière-Lépeaux (1753-1824), instigateur de la création de l'Institut.

Le débat est suivi et animé par des enseignants, des médecins, des vétérinaires, des juristes, des prêtres, c'est-à-dire des membres de la société cultivée, ayant une position pédagogique vis-à-vis du reste de la population, comme le montrent bien les participants au concours de l'Institut ${ }^{16}$ ou ce professeur de l'école vétérinaire de Lyon, dénommé Gronier dans le procès-verbal de la séance. En fait, il s'agit probablement du vétérinaire et agronome Louis-Furcy Grognier (1774-1837), auteur de nombreux ouvrages où transparaissent ses opinions sur le sort

14. Roseline Rey, «L'animalité dans l'œuvre de Bernardin de Saint-Pierre », Revue de synthèse, 113, 1992, p. 31 1-331 ; Colas Duflo, «La zoologie botanique de Bernardin de Saint-Pierre ", Dix-huitième siècle, 42, 2010, p. 385-396, et son édition des Études de la Nature, Saint-Étienne, PU: Saint-Étienne, 2007.

15. Voir les éditions par Jean-Claude Bonnet, Paris, Mercure de France, 1994, et l'édition, par Raymond Trousson, De Jean-Jacques Rousseau considéré comme l'un des premiers auteurs de la révolution, Paris, Champion, 2010. Jean-Claude Bonnet, Louis-Sébastien Mercier, un hérétique en litérature, Paris, Mercure de France, 1995 ; Laurence Mall, "L'Animal et la vérité de l'homme social chez LouisSébastien Mercier ", Dix-huitième siècle, 42, 2010, p. 217-231.

16. Voir la liste très détaillée des mémoires et des professions des candidats dans Valentin Pelosse, "Imaginaire social », op. cit. (n. 11), p. 48-50, avec une prédominance d'enseignants (5), officiers (2), médecins (2), employés de ministère (2), juristes (2), prêtre (1), journaliste (1), pour onze autres, les fonctions ne sont pas indiquées, enfin il y a un adolescent. 
malheureux des bêtes, comme dans ce texte extraordinaire de 1834, où il analyse, en précurseur de l'éthologie, les conséquences pour les vaches de leur spécialisation laitière ${ }^{17}$.

\section{L'INSUPPORTABLE SORT ANIMAL}

Car le point commun de toutes ces prises de parole en temps révolutionnaire est bien la critique du sort habituel des animaux. Critique de la détention de certains à la ménagerie de Versailles, sous l'effet d'un parallèle entre les sujets opprimés et les bêtes encagées. La comparaison était apparue dans la seconde moitié du XVIII ${ }^{e}$ siècle. Barnabé Farmian du Rosoy, partisan d'une monarchie modérée, avait rapproché les cages à oiseaux de la prison de la Bastille et demandé plus de liberté pour les bêtes et les sujets ${ }^{18}$. L'idée culmine sous la Révolution. La municipalité de Paris refuse la présence de montreurs d'animaux lors de la Fête de la Fédération afin de bannir les images d'emprisonnement ${ }^{19}$. Le naturaliste Lacépède soutient en 1795 que les ménageries sont des œuvres de despotes n'ayant « pour la nature que des chaînes à donner ${ }^{20}$. Elles sont des symboles d'oppression de la nature et des bêtes, mais aussi de la société et des sujets. Cette lecture provient de la configuration de la ménagerie versaillaise, construite dans le jardin en 1664 à la demande de Louis XIV. L'entrée s'effectuait par un petit château de plaisir pourvu d'un pavillon octogonal à l'avant, permettant la vue sur une cour de même forme et sur sept enclos d'animaux disposés en éventail autour de cette cour et de ce pavillon. Cette disposition permettait symboliquement à la culture d'enserrer la nature, de rassembler celle-ci autour d'un souverain la dominant et l'embrassant d'un coup d'œil tandis qu'elle s'offrait à lui en lui rendant hommage. "On dirait, écrit Dezallier d'Angeville en 1755, que l'Afrique a payé un tribut de ceux [les animaux] qu'elle produit, et que les autres parties du monde ont fait hommage au roi de ce qu'elles ont de plus rares et de plus singuliers en animaux et en oiseaux. ${ }^{21}$ La ménagerie s'inscrivait dans une affirmation de la

17. Précis d'un cours de multiplication et de perfectionnement des principaux animaux domestiques, Paris, Huzard, 1834. Il publie aussi sur l'hygiène et la zoologie vétérinaires dans ces années 1830 : Précis d'un cours de zoologie vétérinaire, Lyon, Barret, 1833.

18. Les Oiseaux échappés, les paons et l'oiseleur, fable par l'auteur du Cri de l'honneur, s. 1., n. d.

19. Actes de la commune de Paris, Paris, Cerf, 1894-1909, VI, p. 438, 456, 479.

20. Étienne de Lacépède, "Lettre », op. cit.

21. Voyage pittoresque des environs de Paris, Paris, De Bure, 1755, p. 260. 
domination de la nature, mise en œuvre dans toutes les parties du jardin, de l'eau domestiquée en jets d'eau aux arbres taillés en formes géométriques, mais aussi des autres continents, des peuples, et a fortiori des sujets. Ce lien nature-société était bien compris par les contemporains. Il explique la copie de cette ménagerie, au XVIII siècle, par de nombreux princes européens à tendance ou à volonté absolutiste, exprimant ainsi leur désir de domination ${ }^{22}$.

Autre critique, bien plus fréquente : celle des traitements journaliers des animaux domestiques, qualifiés de «barbares" par les membres de l'Institut en 1802, de "crimes " par Gronier en 1803, notamment les violences des rues, les plus signalés par les participants au débat, tous urbains, parce qu'ils en voient sans cesse au long de leurs parcours. Évoquer cette violence pose une question de sources. Jusqu'à la première moitié du $\mathrm{XX}^{e}$ siècle, la plupart des témoignages viennent des bourgeoisies urbaines et accusent des milieux populaires, par exemple les cochers. Du coup, la violence sur l'animal est souvent minorée, voire niée ou négligée par les historiens, les sociologues, les ethnologues parce que ces accusations ne seraient que le fruit d'une lutte des classes ou d'une culture doloriste exacerbée de la part des élites, leur faisant noircir, voire inventer le tableau, et cette position est fortifiée par la gêne croissante à parler de violences populaires. Qu'il y ait un jeu social conduisant ces élites à dénoncer les violences des modestes... en oubliant les leurs, comme la chasse à courre, c'est évident. Que la dénonciation par les premières soit amplifiée par un rejet croissant de la violence physique, alors que les seconds utilisent encore cette violence comme un mode de relation aux autres ${ }^{23}$, pas seulement aux animaux, c'est aussi évident. Cela ne doit pas conduire à nier ou à oublier le phénomène sous prétexte qu'il est noyé dans la subjectivité. Bien sûr, les témoignages sont des filtres qui déforment çà et là, qui inventent quelquefois, mais il faut faire avec en les contrôlant et en les recoupant. D'autre part, il ne faut pas tomber dans le piège de la lecture sociale des sources et prendre le déséquilibre des témoignages pour la preuve d'un clivage social. La violence n'est pas absente des milieux bourgeois, même s'ils en parlent peu. À l'inverse, elle est réprouvée par des modestes lorsqu'ils ne

22. Sur cette lecture politique des ménageries: Éric Baratay, «Le zoo: un lieu politique (XVI'-XIX' siècles) », dans L'Animal en politique, Paul Bacot, Éric Baratay et alii (dir.), Paris, 2003, p. 15-36. Sur l'usage politique des animaux, voir, outre cet ouvrage : Jean Luc Guichet (dir.), Usages politiques de l'animalité, Paris, L'Harmattan, 2008, et Annie Duprat (dir.), Figures animales,
numéro spécial Societés et représentations, 27, 2009, 1.

23. Voir Alain Corbin, Le Village des cannibales, Paris, Aubier, 1992; Frédéric Chauvaud, De Pierre Rivière à Landru, la violence apprivoisée au XIX* siecle, Tournai, Brepols, 1991 ; Id., Les passions villageoises au XIX" siècle, Paris, Publisud, 1995 ; Id. (dir.) Corps saccagés : une histoire des violences corporelles du
siècle des Lumières à nos jours, Rennes, PUR, 2009 . 
partagent pas ou plus les valeurs et les manières qui l'accompagnent. Il faut dépasser le schématique clivage social au profit d'un clivage de sensibilités personnelles ; d'ailleurs, celui-ci est sans cesse pris en compte par les contemporains, par exemple lorsque les compagnies de transport cherchent, et trouvent, des conducteurs « bons » avec leurs chevaux pour améliorer les rendements.

Dans les textes viennent en premier lieu les violences faites aux équidés tirant des voitures, des fiacres, des chariots, et aux chiens de trait, attelés à de petites carrioles : trop gros efforts demandés, trop lourdes charges à tracter, très longs parcours à effectuer, multiples coups reçus pour faire avancer des bêtes très sollicitées, souvent peu nourries, mal soignées. « N'est-il pas choquant, écrit l'un des candidats au concours de 1802, de voir [...] un charretier brutal qui jure d'une manière effroyable et qui déchire à coup de fouet les flancs décharnés de ses chevaux excédés de fatigue et d'inanition. ${ }^{24}$ D'autant que les chevaux travaillent jusqu'à l'épuisement, malgré la douleur et la fatigue, et que leur surmenage peut prendre une forme aiguë extrême, l'animal s'écroulant foudroyé d'une attaque au cœur.

Ce point ouvre un second préalable, celui du niveau de douleur et de souffrance chez les animaux, en particulier les mammiferes concernés par ces gestes et ces discours, notamment les bovins, les chiens, les chevaux. Au risque d'étonner les lecteurs habitués à une vulgate philosophique ressassée, le ressenti de la douleur a été démontrée pour les mammiferes puis les oiseaux au $\mathrm{XX}^{\mathrm{c}}$ siècle, pour des reptiles à la fin des années 1990, des poissons, des mollusques et des crustacés dans les années 2000 . Il a été prouvé qu'il ne s'agissait pas de la simple réaction réflexe inconsciente (nociception), présente chez tous les animaux y compris l'homme, mais bien de douleurs, et que des anatomies et des physiologies très diverses pouvaient assurer ce ressenti douloureux. La proximité physiologique des chevaux, chiens et bovins avec l'homme, notamment la présence d'un néocortex et d'un système de transmission de la douleur, développé, complexe, avec fibres et substances, construit et fonctionnant de la même manière, autorise à dire que leur douleur et leur souffrance sont réelles, semblables et égales en proportion aux humaines. La différence installée dans la précipitation, dans la seconde moitié du $\mathrm{XX}^{\mathrm{e}}$ siècle, entre la douleur physique et la souffrance psychologique, pour différencier l'animal de l'homme, n'a évidemment pas tenu longtemps puisque les vertébrés, notamment les mammifères, ressentent des émotions liées à la douleur (peur, angoisse)

24. Mémoire $n^{\circ} 21$ d'Adrien Gauthier-Lachapelle (Paris, statut non indiqué, désormais s.n.i.). Voir Daniel Roche, La Culture équestre de l'Occident, XVT-XIX' siècle, Paris, Fayard, 2008 ; JeanPierre Digard, Une Histoire du cheval, Arles, Actes Sud, 2007. 
ou tout à fait indépendantes (ennui, solitude, frustration), qu'on peut rassembler sous le terme de souffrance. D'ailleurs, les expressions concrètes sont similaires dans leurs dimensions physiologiques (augmentations cardiaque, respiratoire, hormonale ; sudation; perte de poids), psychologiques (dépression avec apathie ou agressivité, stéréotypies) et comportementales (retraits, vocalises, postures), même s'il y a évidemment des particularités d'espèce, comme les narines et les pupilles dilatées du cheval ou le cou rentré du chien ${ }^{25}$. Si on lit attentivement les textes, en faisant attention aux coups donnés, aux brutalités exercées, et si on analyse cela du côté des bêtes, il apparaît que ces gestes ne sont pas portés au hasard, que les hommes savent comment procéder pour faire souffrir, qu'il s'agit bien d'une réalité et non d'une seule rhétorique, d'un simple sentiment projeté, d'un pur fantasme humain.

D'autre part, la condamnation de ces violences n'est pas seulement due au changement de regard et de sensibilité évoqué plus loin, comme le disent trop facilement les historiens, les ethnologues et les sociologues penchés sur le sujet, parce qu'ils analysent à tort l'animal comme un objet passif, ne réagissant pas, et parce qu'ils ne regardent que le versant humain, examiné trop souvent par l'angle exclusif des lectures culturelles, à la mode depuis vingt ans. Ils oublient ainsi deux réalités : la douleur des bêtes, donc leurs réactions, des cris aux agitations, bien visibles par le public; l'évolution du monde animal, car celui-ci ne constitue pas un décor intangible, éternel, une sorte d'image d'Épinal d'une France d'autrefois. La seconde moitié du XVIII ${ }^{e}$ siècle est marquée par le développement, devenant considérable au $\mathrm{XIX}^{\mathrm{c}}$ siècle, de l'utilisation des animaux domestiques en ville et en campagne, ce qui se traduit par de fortes augmentations des effectifs ${ }^{26}$. Pour le trait, ce sont les chevaux, les mulets, mais aussi les chiens, plus économiques que les équidés. Cette utilisation était bien moindre autrefois, ne serait-ce que par la difficulté à nourrir les bêtes alors que les disettes régnaient fréquemment parmi les hommes. Ces animaux plus nombreux sont maniés par une population n'ayant pas l'habitude

25. Georges Chapoutier, « La douleur : des animaux à l'homme » et Dalila Bovet, "Comment reconnaissons-nous et interprétons-nous les signes extérieurs de la douleur ou de la souffrance des animaux? ", dans Homme et animal : de la douleur à la cruauté, Thierry Auffret Van der Kamp et alit (dir), Paris, 2008, p. 25-38, 47-61 ; A. Branchereau, Ézaluation de la douleur et de l'hyperalgésie chez le bovin, Nantes, Thèse vétérinaire, 2008 ; Caroline Jacques, La Douleur chez le cheval, Lyon, Thèse
vétérinaire, 2001 .

26. Sur ce développement, qui transforme les bêtes, les paysages, et qui bouleverse la société, voir Éric Baratay, La Société des animaux, de la Révolution à la Libération, Paris, La Martinière, 2008 ; Olivier Zeller, "L'Animal dans la ville d'Ancien Régime ", Cahiers d'histoire, 42, 1997, p. 543554 ; Olivier Faure, «Le bétail dans la ville au XIX siècle », Cahiers d'histoire, 42, 1997,
p. 555-573. 
(d'où la multiplication d'accidents avec des bêtes emballées), n'ayant pas la patience, ne cherchant pas à comprendre, reportant la violence vécue dans les rapports sociaux ${ }^{27}$. La condamnation croissante des brutalités est donc aussi une réaction à la multiplication des scènes violentes sur la voie publique du fait de la vulgarisation des usages d'animaux, notamment du transport attelé. Une bonne illustration de cette situation est le développement de la castration des chevaux. Il ne s'agit pas d'une invention de l'époque contrairement à ce que Gronier croit en la condamnant (elle est utilisée au moins depuis l'Antiquité), mais elle connait une forte vulgarisation parce que les nouveaux utilisateurs, vite et mal formés, préfèrent des hongres assagis aux étalons trop impétueux, comme le dénonce ce vétérinaire dans un style oratoire ampoulé, habituel à l'époque : « Renvoyez avec mépris le timide écuyer qui tremble devant son coursier, à moins qu'il ne soit privé des attributs de son sexe. » Or, la castration, effectuée à vif par excision ou écrasement des testicules, est extrêmement douloureuse et provoque une forte mortalité parmi les chevaux opérés ${ }^{28}$.

Autre violence de rue fréquemment condamnée : celle des tueries, pas encore nommées abattoirs, situées dans des cours aux portes ouvertes, débordant fréquemment sur la voie publique par manque de place, et dévoilant ainsi aux passants et aux habitants les terreurs, les cris, les fuites des bêtes, leurs agonies à coups de maillet ou de couteau, le sang et les déchets répandus au sol ${ }^{29}$. « Passant à Montargis, écrit un candidat du concours de 1802, j’aperçus un veau attaché à un poteau par la patte de derrière, ayant celles de devant libres, et se débattant ainsi avec la mort au milieu des souffrances; ce pauvre animal n'avait sans doute reçu qu'un coup de couteau mal appliqué, qui le laissa plus d'un quart d'heure livré à cette torture. Ne pouvant supporter pareil spectacle, j'en fis des reproches amers au boucher qui me répondit froidement que ce n'était qu'un animal. " ${ }^{30}$ Là encore, de tels spectacles se multiplient à l'époque avec le développement de la consommation de viande dans les villes, alors qu'elle avait stagné, même diminué aux $\mathrm{XVII}^{\mathrm{c}}$-XVIII ${ }^{\mathrm{e}}$ siècles, et avec la multiplication des

27. Voir Ghislaine Bouchet, Le Cheval à Paris de 1850 à 1914, Paris, Champion, 1993 ; Patrick Marchand, Le Maitre de poste et le messager : une histoire du transport public en France au temps du chezal, Paris, Belin, 2006 ; Daniel Roche, Daniel Reytier (dir.), Voitures, chevaux et attelages du XVT au $X I X^{r}$ siècle, et $\dot{A}$ cheval! Écuyers, amazones et cavaliers du $X I^{*}$ au XIX siècle, Versailles, Association pour l'Académie d'art équestre de Versailles, 2000 et 2007.

28. Jean-Claude Regnault, "La castration du cheval : aspects historiques ", Pratique vétérinaire équine, 31, 1999, p. 25-28.

29. Sydney Watts, "Boucherie et hygiène à Paris au XVIII siècle ", Revue d'histoire moderne et contemporaine, 51, 2004, 3, p. 79-103 ; Reynald Abad, «Les tueries à Paris sous l'Ancien régime ou pourquoi la capitale n'a pas été dotée d'abattoirs aux XVII'-XVIII siècles ", Histoire, économie et sociétés, 17, 1998, 4, p. 649-676.

30. Mémoire $\mathbf{n}^{\circ} 5$ d'Amable Blanquet (Mende, s.n.i.). 
boucheries. Cela s'accompagne d'acheminements toujours plus nombreux du bétail, avec leur cortège de coups pour faire avancer plus vite des animaux fatigués par le trajet, peu ou pas nourris ni abreuvés parce qu'ils vont mourir ${ }^{31}$.

Les violences d'agrément sont moins citées que les précédentes mais sont tout autant condamnées. La chasse, en pleine expansion avec l'augmentation du nombre des chasseurs, commencée dans la seconde moitié du XVIII' siècle puis amplifiée par l'abolition du privilège aristocratique sous la Révolution ${ }^{32}$, est considérée comme un gaspillage de la nature, notamment lorsqu'elle concerne les oiseaux insectivores utiles à l'agriculture. Les divertissements, comme le jeu de l'oie, dont il faut décapiter ou arracher le cou, et les combats, de coqs, de chiens, de bovins, etc., donnent lieu à des dénonciations dès $1790^{33}$. Ils passionnaient autrefois l'aristocratie, mais elle les a abandonnés au monde populaire, et ils sont multipliés à la fin du XVIII ${ }^{e}$ siècle en conséquence d'un certain enrichissement permettant d'aller voir ces spectacles ou de posséder des bêtes pour en organiser. Bien que peu citée, la vivisection n'en est pas moins condamnée pour les souffrances qu'elle provoque ${ }^{34,}$ d'autant qu'elle a été remise à l'honneur par Lavoisier, travaillant sur le mécanisme de la respiration dans les années 1770-1780, après avoir été lancée par des antiques, tel Galien, puis ressayée un temps par des modernes, tel Harvey au
XVII $^{\mathrm{e}}$ siècle $^{35}$.

Ainsi, la focalisation du débat sur les traitements subis par les animaux n'est pas le fruit du hasard. Elle intervient dans un contexte nouveau d'explosion des utilisations des animaux et des violences à leur égard. Ce n'est donc pas un simple et seul changement de regard, même s'il existe.

31. Mémoire $n^{\circ} 21$, déjà cité. Les doléances révolutionnaires sont confirmées par les traités de médecine vétérinaire ou de zootechnie qui évoquent les pratiques des maquignons. Sur la multiplication du bétail en ville, voir aussi Pierre Olivier Fanica, Le Lait, la vache et le citadin du XVIr au $X X^{\mathrm{c}}$ siècle, Versailles, Quae, 2008.

32. Mémoires $\mathrm{n}^{\circ} 8$ de Christian Warmholz (Rhénanie, enseignant), $\mathrm{n}^{\circ} 17$ de Arbaud (Villeneuve, prêtre). Voir Philippe Salvadori, La Chasse sous l'Ancien Régime, Paris, Fayard, 1996 ; Christian Estève, "Le droit de chasse en France de 1780 à 1914. Conflits d'usages et impasses juridiques ", Histoire et sociétés rurales, 21, 2004, p. 73-114.

33. Mémoires $n^{\circ} 9$ de Dieudonné Malherbe (Liège, s.n.i.), $n^{\circ} 16$ de C. Gaucher (Paris, s.n.i.), $\mathrm{n}^{\circ} 24$ d'Amaury Duval (Paris, chef du bureau des Beaux-Arts). Voir la Gazette nationale, 12 mars,
4 et 18 août 1790 .

34. Mémoire $n^{\circ} 21$, déjà cité.

35. Ce sujet n'est quasiment pas travaillé par les historiens. Alessandro Pastore, « Médecine légale et expérimentation judiciaire : expérimenter le poison sur les animaux en Italie à l'époque
moderne ", Revue d'histoire des sciences humaines, 22, 2010, p. 17-35. 


\section{UNE SENSIBILITÉ NOUVELLE}

En effet, la focalisation du débat intervient à un moment de transformation des représentations et des sensibilités humaines, au moins parmi les milieux cultivés. Les condamnations des mauvais traitements sont prononcées au nom de "l'homme sensible ", présenté comme le nouvel homme à imposer parmi les élites pour remplacer l'homme raisonnable du cartésianisme, accusé de faire preuve de mépris et d'indifférence envers les autres créatures. À l'opposé de cette attitude, l'homme sensible doit agir selon le cœur, faire preuve de sentiments d'humanité et de bienveillance, comme le dit Gronier, parce qu'il ressent dans sa chair la douleur et la souffrance des bêtes, comme l'écrit un candidat du concours de 1802: "Voyez-le cet homme sensible! Tout son intérieur se révolte. Les gémissements de l'animal souffrant sollicitent sa pitié, son œil mourant réclame son secours, et ses membres tremblants sa protection! Un instinct involontaire le rapproche du point de réunion auquel aboutit tout ce qui respire. Il sent la douleur dont il est le témoin ; il frémit, ses entrailles sont déchirées, sa poitrine comprimée, ses nerfs resserrés, et son sang glacé dans ses veines. ${ }^{36}$ Cet homme sensible est un héritage direct des philosophes des Lumières, et surtout de Rousseau souvent cité dans les mémoires de 1802 ou par Gronier, notamment pour sa définition de l'humanité centrée sur l'usage de la pitié et non de la raison.

Ces hommes sensibles refusent la violence sur les animaux pour deux raisons. Il s'agit d'abord de réagir contre la violence en général, quel que soit l'être martyrisé. L'épisode révolutionnaire de la Terreur a joué un rôle déterminant pour les convaincre de la nécessité urgente de discipliner et d'éduquer les populations afin d'éviter le retour d'un tel débordement, d'un tel paroxysme de la violence : "Celui qui ménage à ce point la vie même des animaux, soyez sûrs qu'il respectera le repos, l'honneur, le jour des Citoyens ; qu'il saura, s'il le faut, ce cœur si faible, être mille fois plutôt victime que juge d'un Tribunal Révolutionnaire. $"{ }^{37}$ Car ces hommes sensibles croient en une porosité et une diffusion de la violence; ceux qui l'exercent sur les animaux la porteront un jour sur les hommes ${ }^{38}$. S'occuper des animaux, c'est donc s'occuper des hommes. « Des sages ont frémi en

36. Mémoire $\mathrm{n}^{\circ} 8$, déjà cité.

37. Mémoire ${ }^{\circ} 25$ de J.-S. Delanouë (Porrentruy, enseignant); de même ${ }^{\circ} 3$ de L. R. H. (Lons-le-Saunier, s.n.i.).

38. Mémoires $n^{0} 1$ de Jean-Jacques de Teubern (Ulsen, officier), $n^{\circ} 5$ déjà cité, $n^{\circ} 7$ de Bourguignon-Dumolard (Paris, juge), $\mathbf{n}^{\circ} 21$, déjà cité, $\mathbf{n}^{\circ} 25$ déjà cité. 
considérant jusqu'à quel point les cruautés exercées sur les animaux influent sur la morale publique. Si vous êtes assez heureux pour changer la déplorable destinée des animaux domestiques, vous aurez donc contribué à rendre les hommes meilleurs. " Comme le reconnaît ainsi Gronier, il s'agit d'une vieille idée, exprimée dès l'Antiquité et souvent la seule évoquée par le clergé entre Moyen Âge et Temps modernes pour demander une retenue des hommes envers les animaux $^{39}$. Elle est ici sans cesse mise en avant pour justifier les positions en faveur des bêtes, face aux plus réticents.

Car l'autre raison avancée pour condamner les mauvais traitements est bien plus hétérodoxe à l'époque, même si elle est plus importante pour justifier la compassion envers les animaux. Les auteurs insistent sans cesse sur la sensibilité des bêtes, manifestée par des signes évidents de plaisir, de douleur, de souffrance, au moins celles ayant les mêmes organes aux mêmes fonctions que les hommes et exprimant cette sensibilité de la même manière. Elles excitent directement la pitié $^{40}$. Il existe donc une communauté de la sensibilité, de la souffrance et de l'existence entre les hommes et les animaux, comme l'affirment Gronier ou plusieurs candidats au concours de $1802^{41}$. Ces hommes sensibles prennent le contre-pied d'un Descartes refusant la douleur aux animaux-machines, érigeant la raison en barrière discriminante entre l'homme et les bêtes. La sensibilité et la souffrance unissant les vivants sont plus importantes que la raison séparant pour les cartésiens ou hiérarchisant pour les autres. Les auteurs s'appuient sur Plutarque et Montaigne, pour leur critique de l'inhumanité, sur Condillac, pour celle de Descartes, sur les philosophes des Lumières, dont ils s'affirment les disciples et les propagateurs, notamment, encore une fois, sur Rousseau qui avait élaboré cette notion de communauté de la sensibilité et de la souffrance ${ }^{42}$. Mais il faut analyser ces changements de position d'une autre manière que l'interprétation habituelle. Au regard de la nature animale, bel et bien souffrante, et de la situation nouvelle des animaux à cette époque, ce n'est pas un simple changement de conception au gré des caprices humains à propos d'un objet inerte, mais un désir, une volonté de voir une réalité, souvent occultée pour satisfaire les intérêts humains, à propos d'un acteur réagissant et agissant, et cette volonté émerge aussi à la même époque

39. Voir Éric Baratay, « L'Anthropocentrisme », op. cit. (5), et L'Église et l'Animal, op. cit. (5). professeur).

41. Mémoire $n^{\circ} 4$ de Jean Martin (Caraman, s.n.i.). Voir Éric Pierre, «La souffrance des animaix dans les discours des protecteurs français au XIX ${ }^{\text {e }}$ siècle ", Etudes rurales, 147-148, 1998,
p. 81-97.

42. Thierry Gontier, De l'animal à l'homme, op. cit. (6); François Dagognet, L'Animal selon Condillac. Étude sur le Traité des animaux, Paris, Vrin, 2004 ; Jean-Luc Guichet, Rousseau, op. cit. (6) 
à propos du comportement animal, par exemple chez un CharlesGeorges Leroy, véritable précurseur de l'éthologie ${ }^{43}$. Que cette réalité soit lue par le biais d'un anthropomorphisme, d'importance variable selon les auteurs, est évident, mais cela reste une réalité et il reste une volonté de voir.

\section{LIBÉRER, ÉDUQUER, LÉGIFÉRER}

Condamner ne suffit pas, des mesures s'imposent. Pour les animaux encagés des ménageries, la tentation est grande de prôner leur libération. Des lettrés la demandent, au début de la Révolution, au nom de la sensibilité et de l'humanité de cet âge nouveau, alors que des gravures populaires vantent la liberté en la symbolisant par des lâchers d'oiseaux ${ }^{44}$. En 1793, la municipalité de Paris décide d'interdire l'accès de la ville aux petits montreurs d'animaux et d'envoyer les bêtes confisquées au Jardin des plantes pour les joindre à ceux de la ménagerie de Versailles. Préférant garder les bêtes pour les étudier plutôt que les relâcher, Bernardin de Saint-Pierre et Lacépède proposent d'ériger un parc révolutionnaire pour observer leur comportement, dans le sillage de ce qu'avait prôné Buffon, leur maître, en annonçant l'éthologie du $\mathrm{XX}^{\mathrm{e}}$ siècle ${ }^{45}$. Ce parc serait constitué de vastes enclos garantissant l'intégrité physique des animaux et permettant le déploiement de leurs comportements normaux. Ainsi, écrit Lacépède, les éléphants, les hippopotames, les rhinocéros iraient se baigner sur les berges des canaux et de la Seine, et l'« on pourrait en Europe étudier les habitudes de ces colosses ambulants, dans un état d'indépendance un peu rapprochée de celle dont ils jouissent dans le pays qui les a vus naître ". L'idée préfigure les parcs zoologiques créés en Occident à partir du milieu du $\mathrm{XX}^{\mathrm{e}}$ siècle. Le but n'est pas seulement éthologique mais aussi philosophique, moral, éducatif. Il

43. Lettres sur les animaux, Nuremberg, 1768, et L'Instinct des animaux (1794) réimprimé en L'Intelligence des animaux, Paris, Ibis Press, 2006 ; voir aussi Jean-Claude Bourdin, « L'anthropomorphisme de Charles-Georges Leroy chasseur et philosophe ", Dix-huitième siècle, 42, 2010, p. 353-366.

44. Journal de Paris, 14 juin 1790 ; Gazette nationale, 18 août 1790 ; gravure "Vive la liberté ", publiée par Louise Robbins, Elephant Slaves and Pampered Parrots. Exotic Animals in Eighteenth-Century Paris, London, John Hopkins, 2002, p. 208.

45. Voir Jacques Roger, Buffon, Paris, Fayard, 1989 ; sur l'héritage buffonien : Pietro Corsi, "Buffon sous la Révolution et l'Empire ", dans Buffon 88, J. Gayon (dir.), Paris, 1992, p. 639-648; Jean-Luc Chappey, «L'anthropologie et l'histoire naturelle de l'homme en 1800. Les enjeux d'un héritage », Annales historiques de la Révolution française, 320, 2000, p. 47-54. 
faut, écrit encore Lacépède, que « les images de la contrainte ou les apparences de l'esclavage soient éloignées le plus possible des yeux d'un peuple libre ${ }^{46}$. La libération des bêtes est inscrite dans le sillage des sujets en 1789, des esclaves en 1794 ou des aliénés. Pinel, qui soutient en 1792 la proposition de Bernardin de Saint-Pierre d'un parc d'étude des comportements ${ }^{47}$, transforme ensuite l'hôpital Bicêtre en un lieu d'observations médicales des malades mentaux en les libérant
de leurs chaînes.

Pour les animaux domestiques de tous les jours, des chemins et des rues, la priorité serait d'éduquer les populations des villes et des campagnes avec la diffusion de gravures volantes, prônant les bonnes attitudes, réprouvant les mauvaises, et l'institution de prix récompensant les meilleures conduites et développant l'émulation. Les enfants seraient particulièrement concernés pour éviter qu'ils n'adoptent ou ne développent, selon les conceptions de l'enfance naturellement bonne ou perverse, de mauvais penchants : ils recevraient des leçons et des prix de morale à l'école, celle-ci insérerait le respect de l'animal dans la scolarité ${ }^{48}$. Avec ce primat de l'éducation, repris durant tout le $\mathrm{XIX}^{e}$ siècle et prouvant là encore l'influence des Lumières, en particulier de Rousseau, il s'agirait d'édifier une morale nouvelle, une " morale zootique ", comme l'écrit un candidat de 1802, devant triompher de la cupidité et permettre de vaincre la violence par une "sensibilité en action ${ }^{49}$. Certains arrivent alors à l'idée de devoirs de l'homme, en particulier Gronier qui confie cette tâche aux vétérinaires : ils doivent former les populations et améliorer le sort des
animaux.

Si l'éducation ne suffit pas, une partie des auteurs affirme qu'il faudrait légiférer, voter des lois détaillées et sévères pour les uns, générales et de simple police pour les autres, afin de fermer et d'isoler les abattoirs, de punir les violences et les grossièretés des conducteurs, d'interdire le trait des chiens, les divertissements violents et la vivisection, de supprimer ou au moins de restreindre la chasse, etc. ${ }^{50}$. La

46. Étienne de Lacépède, «Lettre ", op. cit. (9). Sur l'évolution des jardins zoologiques, voir Éric Baratay, Élisabeth Hardouin-Fugier, Zoos, histoire des jardins zoologiques en Occident, XVT-XX" siècles, Paris, La Découverte, 1998 ; Niggel Rothfels, Savages and Beasts: the Birth of Modern Zoo, Baltimore,
Johns Hopkins University Press, From Menagerie to Zoological Park in the Nineteenth Cege, William Deiss (dir.), New World, New Animals. 1996.

47. Aubin Millin, Philippe Pinel, Alexandre Brongniart, Rapport, op. cit. (9)

48. Mémoires $\mathrm{n}^{\circ} 2$ d'Édouard Lambert (Abbeville, s.n.i.), $5,9,20$ déjà cités.

a forgé ce terme de «morale zootique » [voir Valeur de grammaire et de langues anciennes), qui p. 10 , note 7$],$ et $n^{\circ} 7$ déjà cité 50 . Mémoires ñ 3 et 6 déjà
26 de Canolle (Poitiers, médecin). 
violence publique des rues et des chemins est principalement concernée, car elle donne le mauvais exemple, fortifie les mauvais sentiments, contrecarre les actions de sensibilisation. Dans l'optique de sensibilité générale et de morale universelle des auteurs, cette législation devrait faire baisser la violence générale, notamment celle sur les enfants, les femmes ou les vieillards ${ }^{51}$. C'est bien l'idée d'une extension du droit à l'animal qui est ici acquise et prônée publiquement pour la première fois.

Il faut cependant nuancer : la sensibilité, la morale, le respect, le droit concernent les animaux domestiques des campagnes et des villes, ainsi que les animaux sauvages utiles, comme les oiseaux insectivores pour l'agriculture, ou les herbivores pacifiques. Les avis sont souvent hostiles à propos des « vils insectes " qui n'ont pas les « signes connus de la douleur ; ils ne sont pas les représentants convenables de la souffrance ${ }^{52}$. Il est vrai que les cartésiens avaient fait des insectes les modèles de l'animal-machine et cela semble encore évident pour beaucoup d'hommes sensibles, bien que certains tentent de renverser cette représentation en utilisant l'anthropomorphisme, en essayant de se mettre à la place des insectes torturés ${ }^{53}$. Les animaux dits féroces sont encore plus mal lotis. Pour distinguer le nouveau régime politique de celui des souverains aux instincts de chasseurs et de despotes, la ménagerie nationale devrait, écrit Lacépède, écarter ces bêtes parce qu'elles donnent le mauvais exemple de la cruauté dévastatrice, elles font croire que la nature consacre l'empire de la force, elles illustrent et légitiment la tyrannie. Il ne faut que des animaux " innocents et paisibles ", naturellement placés sous la bannière de l'utilité publique, constituant de véritables allégories des citoyens laborieux ${ }^{54}$. Ces animaux paisibles, herbivores, sont promus symboles d'une société travailleuse, pacifiée entre les siens et avec les autres. On retrouve là une idée en vogue parmi les élites cultivées entre 1780 et 1830 ; celle d'un respect, d'un dialogue et d'une communion entre les espèces animales, y compris l'homme, et l'on se passionne pour des histoires d'amitiés interspécifiques, notamment à la nouvelle ménagerie du Jardin des plantes : celle d'un lion et d'un chien en 1794-1796, d'un aigle et d'un coq en 1807, d'une girafe avec son soigneur en $1827^{55}$. Cet espoir est bien illustré par une gravure de l'anglais John Forbes en 1816. Elle représente le Jardin des plantes tel qu'il n'a jamais été, mais tel que

51. Mémoires n ${ }^{\circ} 11,17$ déjà cités.

52. Mémoire $\mathrm{n}^{\circ} 22$ déjà cité.

53. Mémoire $n^{\circ} 21$ déjà cité.

54. Étienne de Lacépède, "Lettre ", op. cit. (9).

55. Georges Toscan, Histoire du lion de la ménagerie [...] et de son chien, Paris, Cuchet, an III ; Magazine pittoresque, 1807, p. 402 ; L. Ferlus, Nouvelle notice sur la girafe, Paris, Moreau, 1827. 
l'artiste souhaiterait apparemment qu'il soit, avec des herbivores (éléphant, zèbre, oiseaux...) libres, vivant ensemble (une utopie mise en scène dans les parcs zoologiques de la seconde moitié $\mathrm{du} \mathrm{XX}^{\mathrm{e}}$ siècle), communiquant entre eux et avec des hommes se promenant parmi
eux dans ce nouvel éden ${ }^{56}$.

\section{UN ÉCHEC PROMETTEUR}

À court terme, rien de tout cela n'est réalisé : ni prix décerné en 1803, ni loi, ni programme d'éducation, ni éden zoologique même s'il y eut une ébauche, avec quelques enclos destinés à des herbivores, mais vite abandonnée au profit de fosses (ours, 1805) et de bâtiments classiques $^{57}$. Le révolutionnaire rocher aux fauves, finalement gardés, est abandonné (il n'est réalisé qu'en 1907 à Stellingen, le premier des zoos contemporain $\mathrm{s}^{58}$ ) et remplacé par une rotonde (1812) composée d'une salle circulaire et de cinq pavillons rayonnants formant les branches d'une croix. Ces fauves sont ensuite installés dans un édifice rectiligne néoclassique, alignant les cages les unes à côté des autres comme la volière des rapaces (1825). La faisanderie (1838) présente un plan radioconcentrique et la singerie (1837), une cage circulaire enserrée par un autre bâtiment néoclassique ${ }^{59}$ !

Au-delà des raisons immédiates, le manque de financement pour le zoo, la qualité jugée insuffisante des mémoires pour le concours ${ }^{60}$, les raisons plus profondes de l'échec sont d'abord politiques : les promoteurs de la ménagerie et du concours de 1802, notamment Bernardin de

56. On trouvera cette gravure dans l'édition anglaise de notre ouvrage: Zoo. A History of Zoological Garden in the West, London, Reaktion Books, 2002, p. 77.

57. Verniquet, Exposition d'un projet sur le Muséum d'histoire naturelle et sur une ménagerie, Paris, Huzard, an X [1801]; AN, AJ 15846 , Programme d'une ménagerie nationale, 27 pluviôse
an X.

fournal of the Society for the "Carl Hagenbeck's Tierpark and Modern Zoological Gardens ", Empire of Entertainments, Washington, Un Natural History 1980, p. 582; Eric Ames, Carl Hagenbeck's Carl Hagenbeck, Hamburg, Ellert \& Richter, 2009.

59 Éric Baratay Elisabeth Harer, 2009.

ménagerie ", op. cit. (8) ; Émilie Châteaun-Fugier, Zoos, op. cit. (n. 46) ; Randy Burkhardt, "La Molinos et la ménagerie du Muséum d'histoire naturelle à dans leur jardin : l'architecte Jacques 2001, p. 75-86 ; Yves Laissus, Les Animaux, op cit (8) àrelle à Paris, 1795-1827 », Histoire de l'art, 49,

60. On peut se demander aussi

des examinateurs, différents de ceux qui ont prope de prix à l'Institut ne vient pas du désintérêt activités. Seul Pierre Samuel Dupon qui ont proposé le sujet et bien éloignés de celui-ci par leurs l'instinct, Paris, impr. de la Revue philosophique, 1806 et d'histoire naturelle, Paris, Delance, 1807, où il accorde un langes mémoires sur diffêrents sujets, la plupart 
Saint-Pierre, mais aussi La Revéllière-Lépeaux, l'abbé Grégoire, LouisSébastien Mercier, sont marginalisés et mis de côté avec la fondation du Consulat puis de l'Empire et le retour d'un pouvoir fort, méfiant envers le bouillonnement intellectuel de la Révolution. À ce propos, une lecture, pourtant sans preuve documentaire, s'impose vite chez des contemporains : l'abandon du parc en liberté au Jardin des plantes aurait été un moyen symbolique d'affirmer un nouveau contrôle de la nature, et par lui de la société. Ainsi, l'architecture de la rotonde, soutenue par le pouvoir impérial et symbolisant bien le changement de conception, est lue, dès les années 1820 semble-t-il, comme une copie de la Légion d'honneur créée en 1802, l'une et l'autre servant à soumettre ${ }^{61}$ !

Plus profondément, l'échec immédiat vient aussi du fait que l'idée d'une domination légitime des hommes sur les bêtes n'est jamais remise en cause par les participants au débat, bien au contraire comme l'illustre Gronier dans son discours, reproduit ci-dessous. Elle prend même une vigueur considérable dès ces débuts du XIX ${ }^{e}$ siècle, en étant fortifiée peu à peu par les "révolutions " agricole et industrielle, les explorations et la colonisation, c'est-à-dire par la maîtrise croissante du monde et de la nature. Tout cela est bien présent lorsqu'un candidat au concours de 1802 propose d'interdire la chasse sauf pour les bêtes féroces, car « elles sont en guerre contre la société, il est donc permis de la leur faire ${ }^{62}$ ou lorsque Gronier propose une attention et un respect utiles, pour mieux exploiter les bêtes, un argument prenant la première place dans le discours de protection au XIX ${ }^{e}$ siècle. D'autre part, si elle permet l'expression politique d'un discours sensible, la Révolution est loin de faire disparaître les autres approches, notamment celle d'inspiration cartésienne, et si les auteurs évoqués tendent à vouloir rehausser l'animal pour le rassembler avec l'homme dans une communauté sensible, d'autres entendent bien les séparer totalement, quitte à dégrader une partie de l'humanité, à qui l'on dénie ce titre, dans une animalité disqualifiée ${ }^{63}$.

Ce n'est pas un hasard si, à partir de cette époque, la philosophie allemande, très influente en France et dans toute l'Europe, de Kant

61. Achille Urbain, «Le parc zoologique du bois de Vincennes », La Revue de Paris, 15 août 1934 , p. 935 , présente cela sous la forme d'une vieille lecture orale au muséum. Sur la raison politique, voir Valentin Pelosse, «Imaginaire social », op. cit. (11); Éric Baratay, « Le zoo : un lieu politique », op. cit. (22).

62. Mémoire $n^{\circ} 17$ déjà cité.

63. Pierre Serna, "Droits d'humanité, droits d'animalité à la fin du $18^{e}$ siècle, ou la matrice du "racisme social" en controverse ", Dix-huitième siècle, 42, 2010, p. 247-263, qui étudie Delamétherie et Jean-Baptiste Salaville, auteur d'un mémoire pour le concours de 1802. Ce cartésien est marginal parmi les auteurs des mémoires du concours et la publication de son livre en 1805, De l'homme, op. cit. (11), déclenche une controverse dans La Décade philosophique: Valentin Pelosse, «Imaginaire social », op. cit. (11), p. 49. 
à Heidegger en passant par Hegel et Marx $^{64}$, impose une définition de l'homme comme sujet agissant dans le monde, le configurant à sa guise, alors que l'animal est considéré privé de monde, prisonnier d'un environnement qu'il ne transformerait pas en raison d'une absence supposée de conscience de soi et de destinée. Il est donc un objet de configuration par l'homme, ce qui exclut considération, respect, devoir, protection, ce qui autorise à en user et en abuser. La constitution de l'homme passe par la destitution de l'animal.

Toutefois, à long terme, cette première révolution intellectuelle annonce et permet les initiatives ultérieures, notamment les premières lois de protection (1824 en Grande-Bretagne, 1850 en France) ${ }^{65}$, dont les motivations et les arguments sont assez semblables aux projets révolutionnaires, ainsi que l'idée de devoir de l'homme, développée dans la seconde moitié du XIX ${ }^{e}$ siècle, puis celles de protection des bêtes sauvages, de sensibilité des animaux, etc. au XX $\mathrm{XX}^{\mathrm{e}}$ siècle. Ce bouillonnement révolutionnaire a été temporaire, mais son importance est analogue à celui qui a lieu au même moment à propos des autres populations, avec la Société des observateurs de l'homme et la Société des amis des noirs.

Du coup, est-ce un hasard si on trouve au croisement de ces deux approches deux personnages importants? L'abbé Grégoire (1750$1831)$, rallié au tiers état en 1789 , rédacteur de propositions de reconnaissance des juifs la même année, de l'égalité civile des Noirs en 1791, puis de l'abolition de l'esclavage, intervenue en 1794, président de la Société des amis des Noirs, auteur De la littérature des nègres en $1808 \ldots$ et membre en 1802 de la section « Morale " de la deuxième classe de l'Institut, celle qui propose le sujet sur les animaux $^{66}$. Bernardin de Saint-Pierre, initiateur de concours du même Institut sur l'éducation des enfants ou les bienfaits de l'abolition de la servitude, et acteur du changement des représentations vis-à-vis des Noirs $^{67}$. Cette liaison est-elle forcée ? Un demi-siècle plus tard, sous la (1).

64. Voir Élisabeth de Fontenay, Le Silence des bêtes, op. cit. (6) ; Jacques Derrida, L'Animal, op. cit.

65. Voir Keath Thomas, Dans le jardin de la nature. La mutation des sensibilités en Angleterre à l'époque moderne, 1500-1800, Paris, Gallimard, 1985 ; Maurice Agulhon, "Le sang des bêtes. Le problème de la protection des animaux en France au XIX ${ }^{e}$ siècle », Romantisme, 31, 1981, p. 81-109 Éric Pierre, "Réformer les relations entre les hommes et les animaux : fonctions et usages de la loi Grammont en France (1850-1914) ", Déviance et société, 31, 2007, p. 65-76.

66. V. Degan, « L'Affirmation des principes de droit naturel par la Révolution française. Le projet de déclaration du droit des gens de l'abbé Grégoire », Annuaire français de droit international, 35, 1989, p. 99-116 ; Yves Bénot, Marcel Dorigny (dir.), Grégoire et la cause des Noirs (1789-1831), Paris, Société française d'histoire d'outre-mer, 2005; Bernard Gainot, "L'abbé Grégoire et la place des Noirs dans l'histoire universelle ", Gradhiva, 10, 2009, p. 22-39.

67. Jean-Charles Pajou (éd.), Esclaves des iles françaises. Lettre sur les Noirs de Bernardin de SaintPierre, Bize-Minervois, Editeurs libres, 2006 ; Bernard Gainot, ibid. 
Deuxième République, Victor Schoelcher fait abolir, en 1848, l'esclavage rétabli par Napoléon, puis soutient ouvertement à l'Assemblée nationale, en 1850, la loi de protection des animaux domestiques sur la voie publique; il est même le député qui intervient le plus à ce propos $^{68}$. Quasiment au même moment, Michelet consacre un chapitre à l'animal dans Le Peuple (1846), en l'insérant entre l'un consacré aux enfants et l'autre aux simples; il s'élève contre les violences faites aux bêtes et compatit à leurs souffrances qu'il voit et admet : " [...] des signes trop visibles expriment ces douleurs, au défaut de langage. Toute la nature proteste contre la barbarie de l'homme qui méconnait, avilit, qui torture son frère inférieur ${ }^{69}$. Belle et bien esquissée sous la Révolution, cette idée de communauté des opprimées est ensuite mise à l'honneur dans la seconde moitié du XIX ${ }^{\mathrm{e}}$ siècle $^{70}$, notamment par $\mathrm{Hugo}^{71}$, Zola ${ }^{72}$, Maupassant ${ }^{73}$, sans doute parce que penser l'autre, c'est un jour penser l'animal.

\section{ANNEXE $^{74}$ :}

\section{Discours de Gronier [Louis-Furcy Grognier], professeur de matière médicale, chimie et botanique, à l'occasion de la remise des prix aux étudiants, imprimé dans le Procès- verbal de la séance publique tenue à l'École vétérinaire de Lyon, le $I^{\text {er }}$ floréal an XI [21 avril 1803] :}

«Je m'adresse à vous, citoyens, qui, par l'étendue et la solidité de vos connaissances, avez été jugés dignes du titre honorable de vétérinaire. Je viens relever à vos yeux la noblesse de votre destination, je viens vous parler des devoirs que vous avez à remplir, des jouissances qui vous sont réservées. Vous êtes encore peu avancés dans la carrière de la vie, tous

68. Le Moniteur universel, 14 juin 1850, p. 2051-2052, 3 juillet 1850, p. 2268-2269. Voir Nelly Schmidt, Victor Schoelcher, Paris, Fayard, 1994 ; Anne Girollet, Victor Schoelcher abolitionniste et républicain, Paris, Karthala, 2000 ; Élisabeth Hardouin-Fugier, «Quelques étapes du droit animalier: Pie V, Schoelcher et Clemenceau », Pouvoirs, 131, 2009, p. 29-4l.

69. Paris, Garnier-Flammarion, 1992, p. 175.

70. Éric Pierre, « La zoophilie dans ses rapports à la philanthropie au XIX ${ }^{e}$ siècle ", Cahiers d'histoire, 42, 1997, p. 655-675; Laurent Fedi, «Pitié pour les animaux : une leçon de morale laique et ses antécédents philosophiques ", Romantisme, 142, 2008, p. 25-40.

71. Claude Millet, "Commençons donc par l'immense pitié" (Victor Hugo) ", Romantisme, 142, 2008, p. 9-23.

72. Émile Zola, « L'Amour des bêtes », Nouvelle campagne - 1896, Paris, Fasquelle, 1897, p. 85-97 ; Danielle Le Blond-Zola, «Émile Zola et l'amour des bêtes », Les Cahiers naturalistes, 30, 1956, p. 284-308.

73. Mont Oriol, Paris, Havard, 1887.

74. Ce procès-verbal, imprimé sous la forme d'une petite brochure volante, est très difficile à trouver dans les archives; nous donnons donc la transcription intégrale du discours de Grognier. 
vos pas seront désormais marqués par des bienfaits rendus à la société. Tantôt vous sauverez la chèvre, unique ressource du pauvre, tantôt vous arrêterez l'épizootie formidable qui menace de désoler tout un empire. Toujours vous trouverez dans votre cour une récompense au-dessus de l'or, au-dessus même de la gloire, le doux, l'auguste sentiment du bien que vous aurez fait.

Vous n'êtes pas seulement appelés à dissiper, à prévenir les maladies qui affligent les animaux domestiques, vous devez encore chercher les moyens d'adoucir leur sort. Combien il est affreux ce sort! Ces êtres sensibles, à qui la mère commune donna droit au bonheur, ont tout perdu en entrant en société avec l'homme, victimes d'un contrat qu'ils n'ont pas consenti, nous récompensons par les traitements les plus barbares, les grands services qu'ils nous rendent.

La justice ne suffit pas pour mettre un frein à la force; la voix de la morale est presque toujours étouffée par celle de la cupidité. Jeunes vétérinaires, parlez le langage de l'intérêt particulier à des hommes qui seraient sourds à celui de la philosophie; prouvez que c'est à son détriment qu'on abuse de sa force, que la cupidité méconnaît presque toujours ses intérêts les plus évidents, que celui qui excède de fatigues le compagnon de ses travaux, qui lui refuse une nourriture suffisante est insensé autant que barbare. C'est ainsi que le sauvage coupe l'arbre pour avoir le fruit, que l'avare de la fable va fouiller dans les entrailles de la poule qui pondait les œufs d'or.

Des sages ont frémi en considérant jusqu'à quel point les cruautés exercées sur les animaux influent sur la morale publique. Si vous êtes assez heureux pour changer la déplorable destinée des animaux domestiques, vous aurez donc contribué à rendre les hommes meilleurs. Le cour, ami de la vertu, palpite à cette idée; oui, l'image du bonheur réfléchit autour de soi par tous les êtres qui se réjouissent de l'existence, dispose l'âme à tous les sentiments d'humanité et de bienveillance. L'habitude au contraire, de voir souffrir dessèche la sensibilité ; elle efface la pitié, la pitié que J.-J. [Jean-Jacques Rousseau] a regardée comme le caractère frappant de la bonté originelle de la nature humaine.

Protecteurs nés des esclaves de l'homme, gardez-vous d'autoriser par votre exemple coupable les atrocités dont les animaux sont les tristes victimes. Refusez le secours de votre bras à l'insensé qui veut arracher à son cheval cet organe que la nature lui donna pour le parer et le défendre. Qu'il ne vous arrive jamais de défigurer bêtement le front le plus noble après le front humain. Renvoyez avec mépris le timide écuyer qui tremble devant son coursier, à moins qu'il ne soit privé des attributs de son sexe. Cette opération ignoble autant que féroce fut, vous ne l'ignorez pas, inconnue à nos pères; vous savez combien elle dégrade, elle avilit le premier des
animaux après l'homme.

Jeunes amis, je n'ajoute qu'un mot, mais qu'il reste profondément gravé dans vos âmes, pénétrez-vous bien que les cruautés exercées sur les animaux sont des crimes, et les services qu'on leur rend de bonnes
actions. " 
Éric Baratay, né en 1960, agrégé d'histoire, habilité à diriger des recherches, professeur d'histoire contemporaine à l'université Jean-Moulin de Lyon depuis 2001, est spécialiste de l'histoire des relations hommes-animaux. Après avoir travaillé sur les représentations (L'Église et l'animal, XVIr-XX' siècle, Le Cerf, 1996) et les pratiques humaines (Zoos, histoire des jardins zoologiques en Occident, $X V T^{\top}-X X^{*}$ siècle, La Découverte, 1998), il s'est intéressé à la condition (Et l'homme créa l'animal, histoire d'une condition, Odile Jacob, 2003) et à la présence sociale des animaux (La Société des animaux, de la Révolution à la Libération, La Martinière, 2008). Il travaille maintenant sur les vécus, les ressentis, les comportements des bêtes (Le Point de oue animal, une autre version de l'Histoire, Le Seuil, à paraître en 2012) de manière à bâtir une éthologie historique et une histoire éthologique, donc une véritable histoire animale.

\section{RÉSUMÉ}

Les débats contemporains sur la sensibilité de l'animal, le respect et la protection de celui-ci, l'extension du droit à son profit et son utilisation éthique sont pour la première fois, non pas évoqués, mais publiquement et politiquement débattus au moment de la Révolution française. Sous l'influence du rousseauisme, prônant la prééminence de la sensibilité sur la raison, et dans le contexte révolutionnaire de bouleversement des idées, des situations et des conditions, cette question des animaux est maintes fois posée dans des rapports, des mémoires et lors de discours officiels. Les thèses avancées et débattues sont étonnement modernes et encore d'actualité. Leur caractère révolutionnaire peut peut-être s'expliquer par la volonté débridée de repenser toutes les conditions, à commencer par les sujets et les esclaves, car penser l'autre c'est un jour penser l'animal.

Mots-clés : Révolution, France, animal, sensibilité, protection.

\section{ABSTRACT}

Animal sensitivity, respect and protection of animals, the expansion of animal rights and the ethical manipulation of animals..., such questions raised today were not only mentioned, but publicly and politically debated for the first time ever under the French Revolution. Inspired by Rousseau, who championed the power of sensibility over reason, the revolutionary context challenged established ideas about the animal condition. A large body of reports, contributions and official speeches reflect the changing perspective men had about other species at the time. Three concrete examples are approached here. The debates about the creation of a menagerie of exotic animals in the Jardin des Plantes of Paris in the 1790s. The competitive examination of 1802 of the class of the moral and political sciences of the National Institute: " up to what point do the barbaric treatments exercised on animals interest the public morality? And would it be advisable to make laws in this respect? ". The speech of a professor of the Veterinary School of Lyon during a session of prize-giving to the students at the end of the study, in 1803. Still today, their reflections on animals are surprisingly modern. Their revolutionary character is due to the unrestrained popular wish to questions all conditions of origin and privilege. For, in the end, looking at "the other " is looking at animals. It is also the will to take into account the pain and the suffering of animals, demonstrated by the current science, guessed and expressed by these revolutionary actors, that the historians have to take into account in their analysis.

Keywords: Revolution, France, Animal, Sensibility, Protection 